Homology, Homotopy and Applications, vol.21(2), 2019, pp.363-375

\title{
PERIODIC PROBLEM ON HOMOTOPY GROUPS OF CHANG COMPLEXES $C_{r}^{n+2, r}$
}

\author{
ZHONGJIAN ZHU, PENGCHENG LI AND JIANZHONG PAN
}

(communicated by John R. Klein)

\begin{abstract}
In this paper, we find new examples, namely one class of Chang complexes $C_{r}^{n+2, r}$, such that their stable homotopy groups are direct summands of their unstable homotopy groups.
\end{abstract}

\section{Introduction}

The study of homotopy groups of finite complexes is one of the central problems in homotopy theory. The main tool for this problem is to decompose its loop space. Serre [12] showed that, $p$-locally for $p$ an odd prime, $\Omega S^{2 n}$ has the homotopy type of the product of $S^{2 n-1}$ and $\Omega S^{4 n-1}$. This decomposition reduces the computation of the odd $p$-primary part of the homotopy groups of spheres to that of odd dimensional spheres. Later Cohen-Moore-Neisendorfer [6] and Cohen [5] found a decomposition of the loop space $\Omega M\left(\mathbb{Z} / p^{r}, n\right)$, where $M\left(\mathbb{Z} / p^{r}, n\right)$ is the Moore space with the only non-trivial homological group $H_{n}\left(M\left(\mathbb{Z} / p^{r}, n\right)\right) \cong \mathbb{Z} / p^{r}$ for some prime $p$ and $p=2$ if $r>1$. One application of these decompositions is determining the exponents of homotopy groups of Moore spaces. Little is known about $\Omega M(\mathbb{Z} / 2, n)$. Recently Selick and $\mathrm{Wu}[\mathbf{9}, \mathbf{1 0}, \mathbf{1 1}]$ developed a functorial decomposition approach to $\Omega \Sigma X$ involving the modular representation theory of symmetric groups. Furthermore, Wu $[\mathbf{1 4}]$ gave another decomposition of $\Omega \Sigma X$ using the free Lie power functor $L_{n}$. One can apply the last decomposition result of Beben-Wu $[\mathbf{1}]$ to find $p$-local (for an odd prime $p$ ) spaces with at most $(p-1)$ cells whose stable homotopy groups are summands of the unstable homotopy groups. Later this result was generalized by Chen and $\mathrm{Wu}[4]$ to the $p=2$ case for 2 -local spaces with 2 cells.

Motivated partly by Selick and Wu's functorial decomposition, the first and third authors [16] studied the decomposability of smash products of indecomposable complexes in $\mathbf{A}_{n}^{2}(n \geqslant 3)$, where $\mathbf{A}_{n}^{k}$ is the homotopy category consisting of $(n-1)$ connected finite $\mathrm{CW}$-complexes with dimension less than or equal to $n+k(n \geqslant$ $k+1$ ). Our next goal is to see whether the stable homotopy groups of indecomposable $\mathbf{A}_{n}^{2}$-complexes (with 3 or 4 cells) are summands of their unstable homotopy groups or not.

The first author was partially supported by National Natural Science Foundation of China (Grant No. 11701430); the second and third authors were partially supported by National Natural Science Foundation of China (Grant No. 11661131004, 11131008).

Received December 11, 2018, revised March 17, 2019, April 14, 2019; published on June 5, 2019. 2010 Mathematics Subject Classification: 55P10, 55P35, 55Q05.

Key words and phrases: Chang-complex, smash product, loop space, decomposition, homotopy group.

Article available at http://dx.doi.org/10.4310/HHA.2019.v21.n2.a20

Copyright (C) 2019, International Press. Permission to copy for private use granted. 
Since the decomposition of the 3 -fold self smash product of any $\mathbf{A}_{n}^{2}$-complex has been completely determined and $\Sigma^{*} C_{r}^{n+2, r}$ is a wedge summand of $L_{3}\left(C_{r}^{n+2, r}\right)$ (while other $\mathbf{A}_{n}^{2}$-complexes with 3 or 4 cells have no such good properties), we concentrate on the study of $C_{r}^{n+2, r}$ in this article. It is helpful to note the difference between Chen and Wu's argument and ours. Their results depend strongly on the assumption that the complexes considered have at most 2 cells when $p=2$. They determined the iterated self smash product of the spaces by using modular representation theory. We have been unable to do the same for Chang complexes by using modular representation theory. Homotopy methods were used successfully to decompose iterated self smash products of Chang complexes and algebraic information from modular representation theory is essential to extract homotopical information on $\Omega \Sigma C_{r}^{n+2, r}$ from the decomposition.

$C_{r}^{n+2, r}(n \geqslant 3)$, one class of elementary Chang complexes, were found by Chang when he classified indecomposable homotopy types in $\mathbf{A}_{n}^{2}(n \geqslant 3)[\mathbf{3}]$.

For stable CW-complexes $X_{i}$ and $Y_{j},(i=1, \ldots, t, j=1, \ldots, s)$, we have the identification:

$$
\left[\bigvee_{i=1}^{t} X_{i}, \bigvee_{j=1}^{s} Y_{j}\right]=\left\{f=\left(f_{k l}\right)=\left(\begin{array}{ccc}
f_{11} & \cdots & f_{1 t} \\
\vdots & \cdots & \vdots \\
f_{s 1} & \cdots & f_{s t}
\end{array}\right): f_{k l} \in\left[X_{l}, Y_{k}\right]\right\}
$$

The cell structures of Elementary Chang complexes are described as follows:

- $C_{\eta}^{n+2}=S^{n} \bigcup_{\eta} \mathbf{C} S^{n+1}$,

- $C^{n+2, s}=\left(S^{n} \vee S^{n+1}\right) \bigcup_{\left(\begin{array}{c}\eta \\ 2^{s}\end{array}\right)} \mathbf{C} S^{n+1}=S^{n} \bigcup_{\eta q} \mathbf{C} M_{2^{s}}^{n}$;

- $C_{r}^{n+2}=S^{n} \bigcup_{\left(2^{r}, \eta\right)} \mathbf{C}\left(S^{n} \vee S^{n+1}\right)=M_{2^{r}}^{n} \bigcup_{i \eta} \mathbf{C} S^{n+1}$;

- $C_{r}^{n+2, s}=\left(S^{n} \vee S^{n+1}\right) \bigcup_{\left(\begin{array}{c}2^{r}, \eta \\ 0,2^{s}\end{array}\right)} \mathbf{C}\left(S^{n} \vee S^{n+1}\right)=\left(M_{2^{r}}^{n} \vee S^{n}\right) \bigcup_{\left(\begin{array}{l}i \eta \\ 2^{s}\end{array}\right)} \mathbf{C} S^{n+1}$

$$
=S^{n} \bigcup_{\left(2^{r}, \eta q\right)} \mathbf{C}\left(S^{n} \vee M_{2^{s}}^{n}\right)=M_{2^{r}}^{n+1} \bigcup_{i \eta q} \mathbf{C} M_{2^{s}}^{n+1}
$$

where $\eta$ is the suspension of the Hopf map, $i$ and $q$ are the canonical inclusion and projection respectively, $n, r, s \in \mathbb{Z}^{+}, n \geqslant 3$ and $M_{p^{r}}^{n}$ denotes $M\left(\mathbb{Z} / p^{r}, n\right)$ for prime $p$.

Chang showed that all indecomposable homotopy types in $\mathbf{A}_{n}^{2}(n \geqslant 3)$ are spheres $S^{n}, S^{n+1}, S^{n+2}$, elementary Moore spaces $M_{p^{r}}^{n}, M_{p^{r}}^{n+1}\left(p\right.$ is a prime, $\left.r \in \mathbb{Z}^{+}\right)$and elementary Chang complexes listed above.

Our main results are given as follows.

Theorem 1.1 (Main theorem). Let $n \geqslant 3$.

$$
\Omega \Sigma C_{r}^{n+2, r} \simeq \prod_{j} \Omega \Sigma C_{r}^{k_{j}(n+1)+1, r} \times(\text { some other space }),
$$

where $2<k_{1}<k_{2}<\cdots$ is a sequence of odd integers such that $k_{j}$ is not a multiple of any $k_{i}$ else for each $j$.

Corollary 1.2. Let $p \geqslant 3$ be an odd integer with $p \geqslant \frac{k-n+1}{n+1}$, then the group $\pi_{k}^{s}\left(C_{r}^{n+3, r}\right)$ is a direct summand of $\pi_{k+(p-1)(n+1)}\left(C_{r}^{n+3, r}\right)$ for $n \geqslant 3$. 
Remark 1.3. From $[\mathbf{1 6}], \pi_{n+2}^{s}\left(C_{r}^{n+3, r}\right) \cong \mathbb{Z} / 2^{r+1}$. Thus from Corollary 1.2 , we get that there is a $\mathbb{Z} / 2^{r+1}$-summand in $\pi_{m}\left(C_{r}^{n+3, r}\right)$ for $m>n+2$ and $m \equiv n+2(\bmod$ $2 n+2)$.

To make the calculations easier to follow, we prove Theorem 1.1 and Corollary 1.2 only for the case $n=3$ in the following sections.

\section{Acknowledgments}

The authors would like to thank Fred Cohen, Jie Wu and Ruizhi Huang for reading an earlier version of this paper and for their many helpful suggestions. We are grateful to the anonymous referee for their careful reading of this paper and, in particular, for pointing out a mistake in a previous proof of Lemma 3.4 .

\section{Preliminaries}

Let $X=\Sigma X^{\prime}$ where $X^{\prime}$ is a path-connected, 2-local finite $C W$-complex, let $n X$ and $X^{\wedge n}$ be the $n$-fold self wedge product and the smash product of $X$, respectively. Let $S_{n}$ denote the symmetric group on $n$ letters and let $\mathbb{Z}_{(2)}\left[S_{n}\right]$ denote the group ring over the 2-local integers $\mathbb{Z}_{(2)}$ generated by $S_{n}$. There is an action of $\mathbb{Z}_{(2)}\left[S_{n}\right]$ on $X^{\wedge n}$ by permuting coordinates and taking the summations. Let $V=\bar{H}_{*}(X ; \mathbb{Z} / 2)$, which is a graded $\mathbb{Z} / 2$-module. For any $\delta \in \mathbb{Z}_{(2)}\left[S_{n}\right]$, there is a map $\delta: X^{\wedge n} \rightarrow X^{\wedge n}$, which induces a map by permuting factors of homologies:

$$
\delta_{*}: V^{\otimes n} \rightarrow V^{\otimes n} .
$$

Start with $\beta_{2}=1-(1,2) \in \mathbb{Z}_{(2)}\left[S_{2}\right]$ and let

$$
\beta_{n}=\beta_{n-1} \wedge i d-(1,2,3, \ldots, n)\left(\beta_{n-1} \wedge i d\right) .
$$

Then for any $x_{1} \otimes x_{2} \otimes \cdots \otimes x_{n} \in V^{\otimes n}$,

$$
\beta_{n *}\left(x_{1} \otimes x_{2} \otimes \cdots \otimes x_{n}\right)=\left[\left[\cdots\left[\left[x_{1}, x_{2}\right], \ldots, x_{n-1}\right], x_{n}\right]\right] .
$$

From [7], for odd $n$, the elements $\frac{1}{n} \beta_{n}$ and $i d-\frac{1}{n} \beta_{n}$ are orthogonal idempotents. Let hocolim $_{f} X^{\wedge n}$ be the mapping telescope of the sequence of maps $X^{\wedge n} \stackrel{f}{\rightarrow} X^{\wedge n} \stackrel{f}{\rightarrow} \cdots$, then $L_{n}(X):=\operatorname{hocolim}_{\frac{1}{n} \beta_{n}} X^{\wedge n}$ is a wedge summand of $X^{\wedge n}$. Let $p_{n}: X^{\wedge n} \rightarrow L_{n}(X)$ be the projection and let $i_{n}: L_{n}(X) \hookrightarrow X^{\wedge n}$ be the canonical inclusion. We have

$$
\left(\frac{1}{n} \beta_{n}\right)_{*}=i_{n *} p_{n *}: H_{*}\left(X^{\wedge n} ; \mathbb{Z} / 2\right) \rightarrow H_{*}\left(X^{\wedge n} ; \mathbb{Z} / 2\right) .
$$

Moreover, from the notation 1.5 of $[\mathbf{1 4}]$

$$
\bar{H}_{*}\left(L_{n}(X) ; \mathbb{Z} / 2\right) \cong L_{n}(V),
$$

where $L_{n}(V)$ is sub-vector space spanned by the Lie elements of tensor length $n$ in the tensor algebra $T(V)$.

Let $1=\Sigma_{\alpha} e_{\alpha}$ be an orthogonal primitive decomposition of the identity element in $\mathbb{Z}_{(2)}\left[S_{n}\right]$ in terms of primitive idempotents. For each $\alpha$, we take $e_{\alpha}(X)=$ 
hocolim $_{e_{\alpha}} X^{\wedge n}$. From $[\mathbf{1 1}, \mathbf{1 5}]$, we have

$$
X^{\wedge n} \simeq \bigvee_{\alpha} e_{\alpha}(X)
$$

For $n=3$, from [8], there is an orthogonal primitive decomposition $1=e_{1}^{\prime}+e_{2}^{\prime}+$ $e_{3}^{\prime}$ of the identity element in $\mathbb{Z} / 2\left[S_{3}\right]$, where $e_{1}^{\prime}=1+\sigma+\sigma^{2}, e_{2}^{\prime}=(1+\tau)(1+\tau \sigma)$, $e_{3}^{\prime}=(1+\tau \sigma)(1+\tau)$ are primitive elements and $\sigma=(1,2,3), \tau=(1,2) \in S_{3}$. It is known that $e_{2}^{\prime}\left(\mathbb{Z} / 2\left[S_{3}\right]\right) \cong e_{3}^{\prime}\left(\mathbb{Z} / 2\left[S_{3}\right]\right)$ and thus $e_{2}^{\prime}, e_{3}^{\prime}$ are related by conjugation in $\mathbb{Z} / 2\left[S_{3}\right]$. By Theorem 1.9.4 of [2], we can lift this decomposition and conjugated relation up to $\mathbb{Z}_{(2)}\left[S_{3}\right]$, i.e., $1=e_{1}+e_{2}+e_{3}$ and there is an element $e \in \mathbb{Z}_{(2)}\left[S_{3}\right]$, such that $e_{3}=e^{-1} e_{2} e$. Hence

$$
X^{\wedge 3} \simeq e_{1}(X) \vee e_{2}(X) \vee e_{3}(X) .
$$

Consider the following maps

$$
e_{2}(X) \stackrel{e_{3} e^{-1}}{\longrightarrow} e_{3}(X) \stackrel{e_{2} e}{\longrightarrow} e_{2}(X) \stackrel{e_{3} e^{-1}}{\longrightarrow} e_{3}(X) .
$$

By $H_{*}\left(e_{i}(X) ; \mathbb{Z}_{(2)}\right)=e_{i *} H_{*}\left(X^{\wedge 3} ; \mathbb{Z}_{(2)}\right)$ for $i=1,2,3$, we get that the composite of two adjacent maps above induces the identity map on $\mathbb{Z}_{(2)}$-coefficient homology groups. Thus

$$
e_{2}(X) \simeq e_{3}(X)
$$

We state the key proposition in the proof of the main theorem, whose proof is given in Section 4.

Proposition 2.1. Let $n \geqslant 3$ be any odd integer, then $L_{n}\left(C_{r}^{5, r}\right)$ has a wedge summand $C_{r}^{4 n+1, r}$.

In order to simplify the writing, let $H_{*}(-)=H(-; \mathbb{Z})$ in the following sections.

\section{Some lemmas on Chang complexes}

Let $v_{n}^{*}, v_{n+1}^{*}, \bar{v}_{n+1}^{*}, v_{n+2}^{*}$ be generators of $\bar{H}^{*}\left(C_{r}^{n+2, r} ; \mathbb{Z} / 2\right)$, which are given in Lemma 3.2 of [16] and denote their dual elements in $H_{*}\left(C_{r}^{n+2, r} ; \mathbb{Z} / 2\right)$ by $v_{n}, v_{n+1}$, $\bar{v}_{n+1}, v_{n+2}$. Note that $S q^{2}\left(v_{n}^{*}\right)=v_{n+2}^{*}$.

Lemma 3.1. If a self-map $f: C_{r}^{n+2, r} \rightarrow C_{r}^{n+2, r}$ induces an automorphism on $H_{n}\left(C_{r}^{n+2, r} ; \mathbb{Z} / 2\right)$ or on $H_{n+1}\left(C_{r}^{n+2, r} ; \mathbb{Z} / 2\right)$, then $f$ is a homotopy equivalence.

Proof. If $f$ induces an automorphism on $H_{n}\left(C_{r}^{n+2, r} ; \mathbb{Z} / 2\right)$, then it induces an automorphism on $H_{n}\left(C_{r}^{n+2, r}\right)$ and $H^{n}\left(C_{r}^{n+2, r} ; \mathbb{Z} / 2\right)$.

Since $H^{n}\left(C_{r}^{n+2, r} ; \mathbb{Z} / 2\right) \stackrel{S q^{2}}{\longrightarrow} H^{n+2}\left(C_{r}^{n+2, r} ; \mathbb{Z} / 2\right)$ is an isomorphism, $f$ induces an automorphism on $H^{n+2}\left(C_{r}^{n+2, r} ; \mathbb{Z} / 2\right)$. From the following commutative diagram

$$
\begin{aligned}
& \operatorname{Ext}\left(H_{n+1}\left(C_{r}^{n+2, r}\right) ; \mathbb{Z} / 2\right) \hookrightarrow H^{n+2}\left(C_{r}^{n+2, r} ; \mathbb{Z} / 2\right) \longrightarrow H o m\left(H_{n+2}\left(C_{r}^{n+2, r}\right), \mathbb{Z} / 2\right)=0 \\
& \operatorname{Ext}\left(f_{*} ; \mathbb{Z} / 2\right) \downarrow \quad f^{*}(\cong) \downarrow \quad \operatorname{Hom}\left(f_{*}, \mathbb{Z} / 2\right) \downarrow \\
& \operatorname{Ext}\left(H_{n+1}\left(C_{r}^{n+2, r}\right) ; \mathbb{Z} / 2\right) \hookrightarrow H^{n+2}\left(C_{r}^{n+2, r} ; \mathbb{Z} / 2\right) \longrightarrow \operatorname{Hom}\left(H_{n+2}\left(C_{r}^{n+2, r}\right), \mathbb{Z} / 2\right)=0
\end{aligned}
$$


we have $\operatorname{Ext}\left(f_{*} ; \mathbb{Z} / 2\right) \neq 0$, which implies that

$$
f_{*}: H_{n+1}\left(C_{r}^{n+2, r}\right)=\mathbb{Z} / 2^{r} \stackrel{\cong}{\rightarrow} H_{n+1}\left(C_{r}^{n+2, r}\right)=\mathbb{Z} / 2^{r} .
$$

So $f$ induces automorphisms on all nontrivial homology groups of $C_{r}^{n+2, r}$; by the Whitehead theorem, $f$ is a self-homotopy equivalence.

If $f$ induces an automorphism on $H_{n+1}\left(C_{r}^{n+2, r} ; \mathbb{Z} / 2\right)$, then it also does on $H^{n+1}\left(C_{r}^{n+2, r} ; \mathbb{Z} / 2\right)$. The conclusion is easily obtained from the following commutative diagram

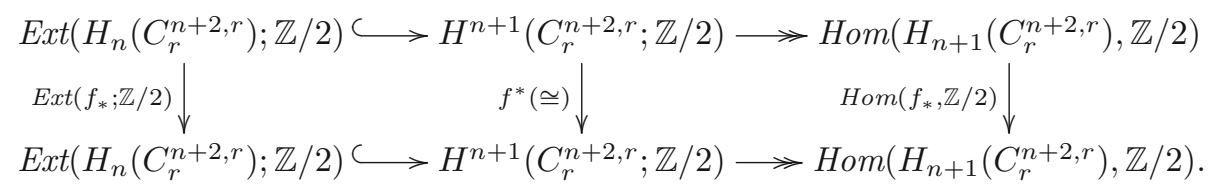

Lemma 3.2. For any map $f: C_{r}^{n+2, r} \rightarrow C_{\eta}^{5} \wedge C_{r}^{n-2, r}(n \geqslant 7)$, $f$ induces a trivial homomorphism

$$
f_{*}=0: H_{n}\left(C_{r}^{n+2, r} ; \mathbb{Z} / 2\right) \rightarrow H_{n}\left(C_{\eta}^{5} \wedge C_{r}^{n-2, r} ; \mathbb{Z} / 2\right) .
$$

Proof. In order to simplify the writing, we prove this lemma for $n=7$.

Suppose that $H_{7}\left(C_{r}^{9, r} ; \mathbb{Z} / 2\right) \stackrel{f_{*}}{\rightarrow} H_{7}\left(C_{\eta}^{5} \wedge C_{r}^{5, r} ; \mathbb{Z} / 2\right)$ is non-trivial, then so is $f^{*}: H^{7}\left(C_{\eta}^{5} \wedge C_{r}^{5, r} ; \mathbb{Z} / 2\right) \rightarrow H^{7}\left(C_{r}^{9, r} ; \mathbb{Z} / 2\right)$. From the following commutative diagram

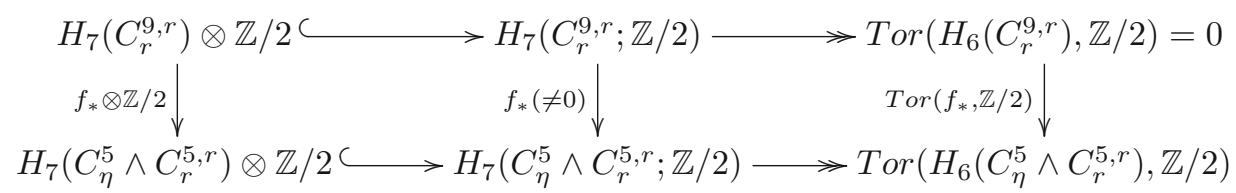

we have $f_{*} \otimes \mathbb{Z} / 2 \neq 0$, which implies that

$$
f_{*}: H_{7}\left(C_{r}^{9, r}\right)=\mathbb{Z} / 2^{r} \cong H_{7}\left(C_{\eta}^{5} \wedge C_{r}^{5, r}\right)=\mathbb{Z} / 2^{r} .
$$

Since $S q^{2}: H^{7}(X ; \mathbb{Z} / 2) \rightarrow H^{9}(X ; \mathbb{Z} / 2)$ is an isomorphism for $X=C_{r}^{9, r}, C_{\eta}^{5} \wedge C_{r}^{5, r}$, we get that $f^{*}: H^{9}\left(C_{\eta}^{5} \wedge C_{r}^{5, r} ; \mathbb{Z} / 2\right) \rightarrow H^{9}\left(C_{r}^{9, r} ; \mathbb{Z} / 2\right)$ is nontrivial.

By the following commutative diagram

$$
\begin{aligned}
& \operatorname{Ext}\left(H_{8}\left(C_{\eta}^{5} \wedge C_{r}^{5, r}\right) ; \mathbb{Z} / 2\right) \hookrightarrow H^{9}\left(C_{\eta}^{5} \wedge C_{r}^{5, r} ; \mathbb{Z} / 2\right) \rightarrow H o m\left(H_{9}\left(C_{\eta}^{5} \wedge C_{r}^{5, r}\right), \mathbb{Z} / 2\right)=\mathbb{Z} / 2 \\
& \operatorname{Ext}\left(f_{*} ; \mathbb{Z} / 2\right) \downarrow \quad f^{*}(\neq 0) \downarrow \quad \operatorname{Hom}\left(f_{*}, \mathbb{Z} / 2\right) \downarrow \\
& \operatorname{Ext}\left(H_{8}\left(C_{r}^{9, r}\right) ; \mathbb{Z} / 2\right) \longleftrightarrow H^{9}\left(C_{r}^{9, r} ; \mathbb{Z} / 2\right) \longrightarrow H o m\left(H_{9}\left(C_{r}^{9, r}\right), \mathbb{Z} / 2\right)=0
\end{aligned}
$$

we have $\operatorname{Ext}\left(f_{*} ; \mathbb{Z} / 2\right) \neq 0$, which implies that

$$
f_{*}: H_{8}\left(C_{r}^{9, r}\right)=\mathbb{Z} / 2^{r} \stackrel{\cong}{\rightarrow} H_{8}\left(C_{\eta}^{5} \wedge C_{r}^{5, r}\right)=\mathbb{Z} / 2^{r} .
$$

It follows that $f^{*}: H^{8}\left(C_{\eta}^{5} \wedge C_{r}^{5, r} ; \mathbb{Z} / 2\right) \rightarrow H^{8}\left(C_{r}^{9, r} ; \mathbb{Z} / 2\right)$ is an isomorphism.

Let $w_{3}^{*}, w_{5}^{*}$ be the generators of $H^{*}\left(C_{\eta}^{5} ; \mathbb{Z} / 2\right)$.

$S q^{2}: H^{6}\left(C_{\eta}^{5} \wedge C_{r}^{5, r} ; \mathbb{Z} / 2\right) \rightarrow H^{8}\left(C_{\eta}^{5} \wedge C_{r}^{5, r} ; \mathbb{Z} / 2\right)$ is non-trivial because $S q^{2}\left(w_{3}^{*} \otimes\right.$ $\left.v_{3}^{*}\right)=w_{3}^{*} \otimes v_{5}^{*}+w_{5}^{*} \otimes v_{3}^{*} \neq 0$. 
Therefore, we get a contradiction by the following commutative diagram

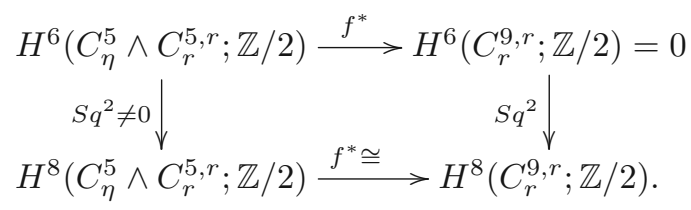

We will use the following cofiber sequences

C1: $C_{r}^{4} \stackrel{2^{r} q_{4}}{\longrightarrow} S^{4} \stackrel{i_{\underline{C}}}{\longrightarrow} C_{r}^{5, r} \stackrel{q_{\underline{C}}}{\longrightarrow} C_{r}^{5} \stackrel{2^{r} q_{5}}{\longrightarrow} S^{5}$;

C2: $S^{3} \stackrel{2^{r} j_{3}}{\longrightarrow} C_{\eta}^{5} \stackrel{i_{C}}{\longrightarrow} C_{r}^{5} \stackrel{q_{C}}{\longrightarrow} S^{4} \stackrel{2^{r} j_{4}}{\longrightarrow} C_{\eta}^{6}$,

which come from the cofiber sequences Cof 5 of $C_{r}^{k, s}$ and Cof $\mathbf{3}$ of $C_{r}^{k}$ in Section 3.2 of [16], respectively.

\section{Lemma 3.3.}

(1) In the following cofiber sequence induced by cofiber sequence $\boldsymbol{C 1}$,

$$
S^{4} \wedge C_{r}^{5, r} \stackrel{i_{\underline{C}} \wedge i d}{\longrightarrow} C_{r}^{5, r} \wedge C_{r}^{5, r} \stackrel{q_{\underline{C}} \wedge i d}{\longrightarrow} C_{r}^{5} \wedge C_{r}^{5, r} \stackrel{2^{r} q_{5} \wedge i d}{\longrightarrow} S^{5} \wedge C_{r}^{5, r}
$$

there is a section $s_{1}: C_{r}^{5} \wedge C_{r}^{5, r} \rightarrow C_{r}^{5, r} \wedge C_{r}^{5, r}$ such that $\left(q_{\underline{C}} \wedge i d\right) s_{1} \simeq i d$.

(2) In the following cofiber sequence induced by cofiber sequence C2,

$$
S^{3} \wedge C_{r}^{5, r} \stackrel{2^{r} j_{3} \wedge i d}{\longrightarrow} C_{\eta}^{5} \wedge C_{r}^{5, r} \stackrel{i_{C} \wedge i d}{\longrightarrow} C_{r}^{5} \wedge C_{r}^{5, r} \stackrel{q_{C} \wedge i d}{\longrightarrow} S^{4} \wedge C_{r}^{5, r}
$$

there is a section $s_{2}: S^{4} \wedge C_{r}^{5, r} \rightarrow C_{r}^{5} \wedge C_{r}^{5, r}$ such that $\left(q_{C} \wedge i d\right) s_{2} \simeq i d$.

Proof. We only prove (1), the proof of (2) is exactly the same as that of (1).

Let $f=i_{C} \wedge i d$ and $g=q_{C} \wedge i d$. Since $\left(i_{C}\right)_{*}$ (resp. $\left.\left(q_{C}\right)_{*}\right)$ is an injection (resp. surjection) in degree 4 homology, so $f_{*}$ (resp. $g_{*}$ ) is an injection (surjection) in degree 7 homology. We have a short exact sequence

$$
0 \rightarrow H_{*}\left(S^{4} \wedge C_{r}^{5, r} ; \mathbb{Z} / 2\right) \stackrel{f_{*}}{\longrightarrow} H_{*}\left(C_{r}^{5, r} \wedge C_{r}^{5, r} ; \mathbb{Z} / 2\right) \stackrel{g_{*}}{\longrightarrow} H_{*}\left(C_{r}^{5} \wedge C_{r}^{5, r} ; \mathbb{Z} / 2\right) \rightarrow 0 .
$$

From Theorem 1.1 of [16], $C_{r}^{5, r} \wedge C_{r}^{5, r} \simeq 2 C_{r}^{9, r} \vee\left(C_{\eta}^{5} \wedge C_{r}^{5, r}\right)$. Let

$$
C_{r}^{5, r} \wedge C_{r}^{5, r} \stackrel{\left(P_{1}, P_{2}, P_{3}\right)^{T}}{\longrightarrow} C_{r}^{9, r} \vee C_{r}^{9, r} \vee\left(C_{\eta}^{5} \wedge C_{r}^{5, r}\right)
$$

where $P_{i}(i=1,2,3)$ are the canonical projections, to the first, second and third wedge summands, respectively; $A^{T}$ is the transpose of a matrix $A$.

By Lemma $3.2, H_{7}\left(P_{3} f ; \mathbb{Z} / 2\right)=0$. Hence there is a map $P=P_{1}$ or $P=P_{2}$, such that $H_{7}(P f ; \mathbb{Z} / 2) \neq 0$. By Lemma 3.1 ,

$$
P f: S^{4} \wedge C_{r}^{5, r} \stackrel{f}{\rightarrow} C_{r}^{5, r} \wedge C_{r}^{5, r} \stackrel{P}{\rightarrow} C_{r}^{9, r}
$$

is a homotopy equivalence.

Let $h:=(P f)^{-1} P: C_{r}^{5, r} \wedge C_{r}^{5, r} \stackrel{P}{\rightarrow} C_{r}^{9, r} \stackrel{(P f)^{-1}}{\longrightarrow} S^{4} \wedge C_{r}^{5, r}$, where $(P f)^{-1}$ is the homotopy inverse of $P f$. Then $h f \simeq i d, h$ is a retraction for $f$; equivalently, there is a section $s_{1}: C_{r}^{5} \wedge C_{r}^{5, r} \rightarrow C_{r}^{5, r} \wedge C_{r}^{5, r}$ such that $\left(q_{\underline{C}} \wedge i d\right) s_{1} \simeq i d$.

Next we study the homotopy type of $L_{3}\left(C_{r}^{5, r}\right)$. 
Lemma 3.4. $L_{3}\left(C_{r}^{5, r}\right) \simeq C_{r}^{13, r} \vee 2\left(C_{\eta}^{5} \wedge C_{r}^{9, r}\right)$.

Proof. $L_{3}\left(C_{r}^{5, r}\right)$ is a wedge summand of $\left(C_{r}^{5, r}\right)^{\wedge 3}$. From Theorem 1.1 of [16], $C_{r}^{5, r} \wedge$ $C_{r}^{5, r} \simeq 2 C_{r}^{9, r} \vee\left(C_{\eta}^{5} \wedge C_{r}^{5, r}\right)$, hence

$$
\left(C_{r}^{5, r}\right)^{\wedge 3} \simeq 4 C_{r}^{13, r} \vee 4\left(C_{\eta}^{5} \wedge C_{r}^{9, r}\right) \vee\left(C_{\eta}^{5} \wedge C_{\eta}^{5} \wedge C_{r}^{5, r}\right),
$$

where $C_{r}^{13, r}$ and $C_{\eta}^{5} \wedge C_{r}^{9, r}$ are indecomposable [16].

From $(2), \operatorname{dim}_{H_{*}}\left(L_{3}\left(C_{r}^{5, r}\right) ; \mathbb{Z} / 2\right)=\operatorname{dim} L_{3}(V)=20$, where $V=\mathbb{Z} / 2\left\langle v_{3}, v_{4}, \bar{v}_{4}, v_{5}\right\rangle$. So we easily get the homology groups of $L_{3}\left(C_{r}^{5, r}\right)$

$$
k=\begin{array}{c|c|c|c|}
10 & \bar{H}_{k}\left(L_{3}\left(C_{r}^{5, r}\right)\right) \\
\hline \bigoplus_{2} \mathbb{Z} / 2^{r} & \bigoplus_{3} \mathbb{Z} / 2^{r} & \bigoplus_{3} \mathbb{Z} / 2^{r} & \underset{2}{\bigoplus} \mathbb{Z} / 2^{r} \\
\hline
\end{array}
$$

Let $Z:=C_{\eta}^{5} \wedge C_{\eta}^{5} \wedge C_{r}^{5, r}$.

All non-trivial reduced homology groups of $Z$ are given as follows:

$$
k=\begin{array}{cc|c|c|c|c|}
9 & 10 & \multicolumn{5}{c}{\bar{H}_{k}\left(C_{\eta}^{5} \wedge C_{\eta}^{5} \wedge C_{r}^{5, r}\right)} \\
\hline \mathbb{Z} / 2^{r} & \mathbb{Z} / 2^{r} & \mathbb{Z} / 2^{r} \oplus \mathbb{Z} / 2^{r} & \mathbb{Z} / 2^{r} \oplus \mathbb{Z} / 2^{r} & \mathbb{Z} / 2^{r} & \mathbb{Z} / 2^{r} \\
\hline
\end{array}
$$

Firstly, we show that if $Z \simeq U \vee V$, with $H_{9} U=\mathbb{Z} / 2^{r}$, then $H_{10} U=\mathbb{Z} / 2^{r}$.

From [15], we have a 2-local homotopy equivalence

$$
C_{\eta}^{5} \wedge C_{\eta}^{5} \wedge C_{\eta}^{5} \simeq 2 C_{\eta}^{13} \vee\left(C_{\eta}^{5} \wedge C_{\nu}^{10}\right)
$$

where $C_{\nu}^{10}=\Sigma^{2} \mathbb{H} P^{2}$, with $S q^{4}: H^{6}\left(C_{\nu}^{10} ; \mathbb{Z} / 2\right) \stackrel{\cong}{\rightarrow} H^{10}\left(C_{\nu}^{10} ; \mathbb{Z} / 2\right)$.

Thus

$$
C_{\eta}^{5} \wedge C_{\eta}^{5} \wedge C_{\eta}^{5} \wedge C_{r}^{5, r} \simeq 2\left(C_{\eta}^{13} \wedge C_{r}^{5, r}\right) \vee\left(C_{\eta}^{5} \wedge C_{\nu}^{10} \wedge C_{r}^{5, r}\right)
$$

The nontrivial reduced homology groups of $L=C_{\eta}^{5} \wedge C_{\nu}^{10} \wedge C_{r}^{5, r}$ are given as follows

$$
k=\begin{array}{cc|c|c|c|c|c|c|}
\multicolumn{8}{c}{\bar{H}_{k}\left(C_{\eta}^{5} \wedge C_{\nu}^{10} \wedge C_{r}^{5, r}\right)} \\
12 & 13 & 14 & 15 & 16 & 17 & 18 & 19 \\
\hline \mathbb{Z} / 2^{r} & \mathbb{Z} / 2^{r} & \mathbb{Z} / 2^{r} & \mathbb{Z} / 2^{r} & \mathbb{Z} / 2^{r} & \mathbb{Z} / 2^{r} & \mathbb{Z} / 2^{r} & \mathbb{Z} / 2^{r} \\
\hline
\end{array}
$$

(Sq1) $0 \rightarrow H^{12}(L ; \mathbb{Z} / 2) \stackrel{S q^{6}}{\longrightarrow} H^{18}(L ; \mathbb{Z} / 2) \stackrel{S q^{2}}{\longrightarrow} H^{20}(L ; \mathbb{Z} / 2) \rightarrow 0$ is exact;

(Sq2) $H^{12}(L ; \mathbb{Z} / 2) \stackrel{S q^{4}}{\longrightarrow} H^{16}(L ; \mathbb{Z} / 2)$ is injective;

(Sq3) $H^{12}(L ; \mathbb{Z} / 2) \stackrel{S q^{8}}{\longrightarrow} H^{20}(L ; \mathbb{Z} / 2)$ is an isomorphism.

$C_{\eta}^{5} \wedge C_{\eta}^{5} \wedge C_{\eta}^{5} \wedge C_{r}^{5, r}$ and $C_{\eta}^{13} \wedge C_{r}^{5, r}$ are self dual under Spanier-Whitehead-Duality $D_{32}: \mathbf{A}_{12}^{8} \rightarrow \mathbf{A}_{12}^{8}$, so is $L$.

Suppose $L \simeq X \vee Y$ with $X$ indecomposable and $H_{12} X=\mathbb{Z} / 2^{r}$, then $H^{12}(X ; \mathbb{Z} / 2)$ $\cong H^{12}(L ; \mathbb{Z} / 2)=\mathbb{Z} / 2$. By $(\mathrm{Sq} 3), H^{20}(X ; \mathbb{Z} / 2) \cong H^{20}(L ; \mathbb{Z} / 2)=\mathbb{Z} / 2$, and thus $H_{19} X=$ $\mathbb{Z} / 2^{r}$. From $(\mathrm{Sq} 1), H^{18}(X ; \mathbb{Z} / 2) \cong H^{18}(L ; \mathbb{Z} / 2) \cong \mathbb{Z} / 2 \oplus \mathbb{Z} / 2$. Hence $H_{17} X \cong H_{18} X=$ $\mathbb{Z} / 2^{r}$. Since $L$ is self dual and $X$ contains both the bottom and top cells of $L, X$ is also self dual. We have $H_{13} X \cong H_{14} X=\mathbb{Z} / 2^{r}$. From (Sq2), $H_{15} X \oplus H_{16} X \neq 0$, hence $H_{15} X \cong H_{16} X=\mathbb{Z} / 2^{r}$ by self duality of $X$. Thus $\bar{H}_{m}(Y)=0$ for any $m$, which implies that $Y$ is contractible. Thus we get $L$ is indecomposable. 
$C_{\eta}^{5} \wedge C_{\eta}^{5} \wedge C_{\eta}^{5} \wedge C_{r}^{5, r} \simeq\left(C_{\eta}^{5} \wedge U\right) \vee\left(C_{\eta}^{5} \wedge V\right) \simeq 2\left(C_{\eta}^{13} \wedge C_{r}^{5, r}\right) \vee\left(C_{\eta}^{5} \wedge C_{\nu}^{10} \wedge C_{r}^{5, r}\right)$

Since $C_{\eta}^{13} \wedge C_{r}^{5, r}$ and $C_{\eta}^{5} \wedge C_{\nu}^{10} \wedge C_{r}^{5, r}$ are indecomposable and by observing the bottom cells, we get $C_{\eta}^{5} \wedge C_{\nu}^{10} \wedge C_{r}^{5, r}$ is a wedge summand of $C_{\eta}^{5} \wedge U$, hence $C_{\eta}^{5} \wedge V$ is homotopy equivalent to $C_{\eta}^{13} \wedge C_{r}^{5, r}$ or $2 C_{\eta}^{13} \wedge C_{r}^{5, r}$. By the Künneth formula, $H_{10} V=0$. Hence $H_{10} U=\mathbb{Z} / 2^{r}$.

From the analysis above, the nontrivial 10-dimensional homology groups of $L_{3}\left(C_{r}^{5, r}\right)$ must come from the wedge summand $4\left(C_{\eta}^{5} \wedge C_{r}^{9, r}\right)$ of $\left(C_{r}^{5, r}\right)^{\wedge 3}$. So $L_{3}\left(C_{r}^{5, r}\right) \simeq 2\left(C_{\eta}^{5} \wedge\right.$ $\left.C_{r}^{9, r}\right) \vee Q$, where $Q$ is a space whose only nontrivial homology groups are $H_{11} Q \cong$ $H_{12} Q \cong \mathbb{Z} / 2^{r}$. Since the sequence $H^{10}(Z ; \mathbb{Z} / 2) \stackrel{S q^{2}}{\longrightarrow} H^{12}(Z ; \mathbb{Z} / 2) \stackrel{S q^{2}}{\longrightarrow} H^{14}(Z ; \mathbb{Z} / 2)$ is exact, the Moore spaces $M_{2^{r}}^{11}$ and $M_{2^{r}}^{12}$ cannot be wedge summands of $Z$. Hence $Q$ is not equivalent to $M_{2^{r}}^{11} \vee M_{2^{r}}^{12}$. By the classification of indecomposable complexes in $\mathbf{A}_{n}^{2}(n>3), Q \simeq C_{r}^{13, r}$. This finishes the proof of Lemma 3.4.

Define a map

$$
\phi: C_{r}^{13, r} \simeq S^{4} \wedge S^{4} \wedge C_{r}^{5, r} \rightarrow C_{r}^{5, r} \wedge C_{r}^{5, r} \wedge C_{r}^{5, r}
$$

by $\phi=\left(i d \wedge s_{1} s_{2}\right)(T \wedge i d)\left(i d \wedge i_{\underline{C}} \wedge i d\right)$, where $T(X \wedge Y)=Y \wedge X$. Then $\phi_{*}\left(\iota_{4} \otimes \iota_{4} \otimes\right.$ $u)=v_{4} \otimes \bar{v}_{4} \otimes u$ for $u \in\left\{v_{3}, v_{4}, \bar{v}_{4}, v_{5}\right\}$, where $\phi_{*}$ is the induced map of $\phi$ on $\mathbb{Z} / 2$ homology groups and $\iota_{n}$ is the generator of $\bar{H}_{*}\left(S^{n} ; \mathbb{Z} / 2\right)$.

Lemma 3.5. The composition map

$$
h:=\bar{p} p_{3} \phi: C_{r}^{13, r} \stackrel{\phi}{\rightarrow}\left(C_{r}^{5, r}\right)^{\wedge 3} \stackrel{p_{3}}{\longrightarrow} L_{3}\left(C_{r}^{5, r}\right) \simeq C_{r}^{13, r} \vee 2\left(C_{\eta}^{5} \wedge C_{r}^{9, r}\right) \stackrel{\bar{p}}{\rightarrow} C_{r}^{13, r}
$$

is a homotopy equivalence, where $\bar{p}$ is the canonical projection.

Proof. Note that $H_{*}\left(C_{r}^{13, r} ; \mathbb{Z} / 2\right) \stackrel{\left(p_{3} \phi\right)_{*}}{\longrightarrow} H_{*}\left(L_{3}\left(C_{r}^{5, r}\right) ; \mathbb{Z} / 2\right) \stackrel{i_{3 *}}{\longrightarrow} H_{*}\left(\left(C_{r}^{5, r}\right)^{\wedge 3} ; \mathbb{Z} / 2\right)$ takes $\iota_{4} \otimes \iota_{4} \otimes u$ to $\left[\left[v_{4}, \bar{v}_{4}\right], u\right]$ for $u \in\left\{v_{3}, v_{4}, \bar{v}_{4}, v_{5}\right\}$, hence $i_{3 *}\left(p_{3} \phi\right)_{*}$ is a monomorphism, which implies that $\left(p_{3} \phi\right)_{*}$ is a monomorphism. From Lemma 3.2, for any wedge summand $C_{\eta}^{5} \wedge C_{r}^{9, r}$ of $L_{3}\left(C_{r}^{5, r}\right)$, the map $f=\bar{p}_{2} p_{3} \phi$ induces the trivial homomorphism

$$
f_{*}: H_{11}\left(C_{r}^{13, r} ; \mathbb{Z} / 2\right) \rightarrow H_{11}\left(C_{\eta}^{5} \wedge C_{r}^{9, r} ; \mathbb{Z} / 2\right),
$$

where $L_{3}\left(C_{r}^{5, r}\right) \simeq C_{r}^{13, r} \vee\left(C_{\eta}^{5} \wedge C_{r}^{9, r}\right) \stackrel{\bar{p}_{2}}{\longrightarrow} C_{\eta}^{5} \wedge C_{r}^{9, r}$ is the canonical projection to this wedge summand.

Then the lemma is easily obtained from Lemma 3.1.

\section{Proof of Proposition 2.1}

Let

$$
\begin{aligned}
& \phi_{1}:=i_{3} p_{3} \phi: C_{r}^{13, r} \simeq S^{4} \wedge S^{4} \wedge C_{r}^{5, r} \stackrel{\phi}{\rightarrow}\left(C_{r}^{5, r}\right)^{\wedge} \stackrel{p_{3}}{\longrightarrow} L_{3}\left(C_{r}^{5, r}\right) \stackrel{i_{3}}{\longrightarrow}\left(C_{r}^{5, r}\right)^{\wedge 3} \\
& \varphi_{1}:=h^{-1} \bar{p} p_{3}:\left(C_{r}^{5, r}\right)^{\wedge 3} \stackrel{p_{3}}{\longrightarrow} L_{3}\left(C_{r}^{5, r}\right) \stackrel{\bar{p}}{\rightarrow} C_{r}^{13, r} \stackrel{h^{-1}}{\longrightarrow} C_{r}^{13, r},
\end{aligned}
$$

where $h^{-1}$ is the homotopy inverse of $h$ in Lemma 3.5. So

$$
\varphi_{1} \phi_{1}=h^{-1} \bar{p} p_{3} i_{3} p_{3} \phi=h^{-1} \bar{p} p_{3} \phi=h^{-1} h \simeq i d .
$$


Let $\phi_{k}=i_{2 k+1} p_{2 k+1}\left(\phi_{1} \wedge i d\right)\left(i d \wedge \phi_{k-1}\right)$, i.e., the composite of the following maps

$$
\begin{aligned}
C_{r}^{8 k+5, r} \simeq\left(S^{4}\right)^{\wedge 2} \wedge C_{r}^{8 k-3, r} & \stackrel{i d \wedge \phi_{k-1}}{\longrightarrow}\left(S^{4}\right)^{\wedge 2} \wedge\left(C_{r}^{5, r}\right)^{\wedge 2 k-1}=C_{r}^{13, r} \wedge\left(C_{r}^{5, r}\right)^{\wedge 2 k-2} \\
& \stackrel{\phi_{1} \wedge i d}{\longrightarrow}\left(C_{r}^{5, r}\right)^{\wedge 2 k+1} \stackrel{p_{2 k+1}}{\longrightarrow} L_{2 k+1}\left(C_{r}^{5, r}\right) \stackrel{i_{2 k+1}}{\longrightarrow}\left(C_{r}^{5, r}\right)^{\wedge 2 k+1},
\end{aligned}
$$

and $\varphi_{k}=\left(i d \wedge \varphi_{1}\right)\left(\varphi_{k-1} \wedge i d\right)$, i.e., the composite of the following maps

$$
\begin{aligned}
\left(C_{r}^{5, r}\right)^{\wedge 2 k+1}=\left(C_{r}^{5, r}\right)^{\wedge 2 k-1} \wedge\left(C_{r}^{5, r}\right)^{\wedge 2} \stackrel{\varphi_{k-1} \wedge i d}{\longrightarrow}\left(S^{4}\right)^{\wedge 2 k-2} \wedge\left(C_{r}^{5, r}\right)^{\wedge 3} & \stackrel{i d \wedge \varphi_{1}}{\longrightarrow}\left(S^{4}\right)^{\wedge 2 k} \wedge C_{r}^{5, r} \simeq C_{r}^{8 k+5} .
\end{aligned}
$$

Proof of Proposition 2.1. Since $i_{2 k+1} p_{2 k+1}$ factors through $L_{2 k+1}\left(C_{r}^{5, r}\right), C_{r}^{8 k+5, r}$ is a wedge summand of $L_{2 k+1}\left(C_{r}^{5, r}\right)$ is a direct corollary of Lemma 4.1.

Lemma 4.1. $\varphi_{k} \phi_{k}$ is a homotopy equivalence.

Proof. By Lemma 3.1, it suffices to show that $\varphi_{k *} \phi_{k *}$ on $H_{8 k+3}\left(C_{r}^{8 k+5, r} ; \mathbb{Z} / 2\right)$ is an automorphism:

$$
C_{r}^{8 k+5, r} \simeq\left(S^{4}\right)^{\wedge 2 k} \wedge C_{r}^{5, r} \stackrel{\varphi_{k} \phi_{k}}{\longrightarrow} C_{r}^{8 k+5, r} \simeq\left(S^{4}\right)^{\wedge 2 k} \wedge C_{r}^{5, r} .
$$

Actually, we shall prove that $\varphi_{k *} \phi_{k *}\left(\iota_{4}^{\otimes 2 k} \otimes v_{3}\right)=\iota_{4}^{\otimes 2 k} \otimes v_{3}$ by induction.

From (1), $\varphi_{1} \phi_{1} \simeq i d$.

For $C_{r}^{8 k-3, r} \simeq\left(S^{4}\right)^{\wedge 2 k-2} \wedge C_{r}^{5, r} \stackrel{\varphi_{k-1} \phi_{k-1}}{\longrightarrow} C_{r}^{8 k-3, r} \simeq\left(S^{4}\right)^{\wedge 2 k-2} \wedge C_{r}^{5, r}$, assume that the induced endomorphism $\varphi_{k-1 *} \phi_{k-1 *}$ on $H_{*}\left(C_{r}^{8 k-3, r} ; \mathbb{Z} / 2\right)$ is the identity on generators $\iota_{4}^{\otimes 2 k-2} \otimes v_{3} \in H_{8 k-5}\left(\left(S^{4}\right)^{\wedge 2 k-2} \wedge C_{r}^{5, r} ; \mathbb{Z} / 2\right)$.

Denote by $a d(x)(y)=[y, x]$ and $a d^{i+1}(x)(y)=\left[a d^{i}(x)(y), x\right]$ for $i \geqslant 1$. The coefficients are taken mod-2, hence $[u, v]=[v, u]$ and $\operatorname{ad}([u, v])(u)=[[u, v], u]=[u,[u, v]]$.

\section{Claim 1.}

(i) $\beta_{3 *}([x, y] \otimes z)=0 ; \beta_{3 *}(z \otimes[x, y])=[[x, y], z]$; $\beta_{3 *}([[x, y], z])=[[x, y], z]$,

(ii) $\beta_{2 k+1 *}([[x, y], x] \otimes y \otimes w)+\beta_{2 k+1 *}([[x, y], y] \otimes x \otimes w)=0$,

where $x, y, z \in \bar{H}_{*}(X ; \mathbb{Z} / 2)$ and $w \in \bar{H}_{*}\left(X^{\wedge 2 k-3} ; \mathbb{Z} / 2\right)$.

Proof of Claim 1. (i) of Claim 1 is obvious. We prove (ii) of Claim 1 in the following.

$[[x, y], x] \otimes y+[[x, y], y] \otimes x$

$$
=x \otimes x \otimes y \otimes y+y \otimes x \otimes x \otimes y+y \otimes y \otimes x \otimes x+x \otimes y \otimes y \otimes x .
$$

Note that $\beta_{2 k+1 *}(x \otimes x \otimes y \otimes y \otimes w)$ and $\beta_{2 k+1 *}(y \otimes y \otimes x \otimes x \otimes w)$ are zero. By

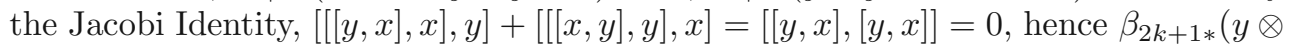
$x \otimes x \otimes y \otimes w)+\beta_{2 k+1 *}(x \otimes y \otimes y \otimes x \otimes w)=0$. Thus we complete the proof (ii) of Claim 1.

Claim 2. $\beta_{2 k+1 *}\left(\left[\left[v_{4}, \bar{v}_{4}\right], v_{3}\right] \otimes\left[v_{4}, \bar{v}_{4}\right]^{\otimes k-1}\right)=a d^{k}\left(\left[v_{4}, \bar{v}_{4}\right]\right)\left(v_{3}\right)$ for $k \geqslant 1$.

Proof of Claim 2. We prove Claim 2 by induction on $k$.

For $k=1, \beta_{3 *}\left(\left[\left[v_{4}, \bar{v}_{4}\right], v_{3}\right]\right)=\left[\left[v_{4}, \bar{v}_{4}\right], v_{3}\right]=\operatorname{ad}\left(\left[v_{4}, \bar{v}_{4}\right]\right)\left(v_{3}\right)$. 
Assume that $\beta_{2 k-1 *}\left(\left[\left[v_{4}, \bar{v}_{4}\right], v_{3}\right] \otimes\left[v_{4}, \bar{v}_{4}\right]^{\otimes k-2}\right)=a d^{k-1}\left(\left[v_{4}, \bar{v}_{4}\right]\right)\left(v_{3}\right)$. Then

$$
\begin{aligned}
\beta_{2 k+1 *}\left([ [ v _ { 4 } , \overline { v } _ { 4 } ] , v _ { 3 } ] \otimes \left[v_{4},\right.\right. & \left.\left.\bar{v}_{4}\right]^{\otimes k-1}\right) \\
= & \beta_{2 k+1 *}\left(\left[\left[v_{4}, \bar{v}_{4}\right], v_{3}\right] \otimes\left[v_{4}, \bar{v}_{4}\right]^{\otimes k-2} \otimes v_{4} \otimes \bar{v}_{4}\right) \\
& +\beta_{2 k+1 *}\left(\left[\left[v_{4}, \bar{v}_{4}\right], v_{3}\right] \otimes\left[v_{4}, \bar{v}_{4}\right]^{\otimes k-2} \otimes \bar{v}_{4} \otimes v_{4}\right) \\
= & {\left[\left[\beta_{2 k-1 *}\left(\left[\left[v_{4}, \bar{v}_{4}\right], v_{3}\right] \otimes\left[v_{4}, \bar{v}_{4}\right]^{\otimes k-2}\right), v_{4}\right], \bar{v}_{4}\right] } \\
& +\left[\left[\beta_{2 k-1 *}\left(\left[\left[v_{4}, \bar{v}_{4}\right], v_{3}\right] \otimes\left[v_{4}, \bar{v}_{4}\right]^{\otimes k-2}\right), \bar{v}_{4}\right], v_{4}\right] \\
= & {\left[\left[a d^{k-1}\left(\left[v_{4}, \bar{v}_{4}\right]\right)\left(v_{3}\right), v_{4}\right], \bar{v}_{4}\right]+\left[\left[a d^{k-1}\left(\left[v_{4}, \bar{v}_{4}\right]\right)\left(v_{3}\right), \bar{v}_{4}\right], v_{4}\right] } \\
= & {\left[\left[a d^{k-1}\left(\left[v_{4}, \bar{v}_{4}\right]\right)\left(v_{3}\right),\left[v_{4}, \bar{v}_{4}\right]\right]\right] \quad(\text { Jacobi's identity)}} \\
= & a d^{k}\left(\left[v_{4}, \bar{v}_{4}\right]\right)\left(v_{3}\right) .
\end{aligned}
$$

We complete the proof of Claim 2.

Claim 3. $\phi_{k *}\left(\iota_{4}^{\otimes 2 k} \otimes v_{3}\right)=a d^{k}\left(\left[v_{4}, \bar{v}_{4}\right]\right)\left(v_{3}\right)$.

Proof of Claim 3. We prove Claim 3 by induction on $k$.

$$
\phi_{1}\left(\iota_{4} \otimes \iota_{4} \otimes v_{3}\right)=\left[\left[v_{4}, \bar{v}_{4}\right], v_{3}\right]=\operatorname{ad}\left(\left[v_{4}, \bar{v}_{4}\right]\right)\left(v_{3}\right) .
$$

Assume that $\phi_{k-1 *}\left(\iota_{4}^{\otimes 2 k-2} \otimes v_{3}\right)=a d^{k-1}\left(\left[v_{4}, \bar{v}_{4}\right]\right)\left(v_{3}\right)$.

$$
\begin{aligned}
\phi_{k *}\left(\iota_{4}^{\otimes 2 k} \otimes v_{3}\right)= & i_{2 k+1 *} p_{2 k+1_{*}}\left(\phi_{1} \wedge i d\right)_{*}\left(i d \otimes \phi_{k-1 *}\right)\left(\iota_{4}^{\otimes 2} \otimes \iota_{4}^{\otimes 2 k-2} \otimes v_{3}\right) \\
= & \beta_{2 k+1 *}\left(\phi_{1} \wedge i d\right)_{*}\left(\iota_{4}^{\otimes 2} \otimes a d^{k-1}\left(\left[v_{4}, \bar{v}_{4}\right]\right)\left(v_{3}\right)\right) \\
= & \beta_{2 k+1 *}\left(\phi_{1} \wedge i d\right)_{*}\left(\iota_{4}^{\otimes 2} \otimes\left[v_{4}, \bar{v}_{4}\right] \otimes a d^{k-2}\left(\left[v_{4}, \bar{v}_{4}\right]\right)\left(v_{3}\right)\right) \\
& +\beta_{2 k+1 *}\left(\phi_{1} \wedge i d\right)_{*}\left(\iota_{4}^{\otimes 2} \otimes a d^{k-2}\left(\left[v_{4}, \bar{v}_{4}\right]\right)\left(v_{3}\right) \otimes\left[v_{4}, \bar{v}_{4}\right]\right)
\end{aligned}
$$

\section{Observation A.}

$$
\begin{aligned}
\beta_{2 k+1 *}\left(\phi_{1} \wedge i d\right)_{*}\left(\iota_{4}^{\otimes 2} \otimes\left[v_{4}, \bar{v}_{4}\right] \otimes\right. & \left.a d^{k-2}\left(\left[v_{4}, \bar{v}_{4}\right]\right)\left(v_{3}\right)\right) \\
= & \beta_{2 k+1 *}\left(\phi_{1} \wedge i d\right)_{*}\left(\iota_{4}^{\otimes 2} \otimes v_{4} \otimes \bar{v}_{4} \otimes a d^{k-2}\left(\left[v_{4}, \bar{v}_{4}\right]\right)\left(v_{3}\right)\right) \\
& +\beta_{2 k+1 *}\left(\phi_{1} \wedge i d\right)_{*}\left(\iota_{4}^{\otimes 2} \otimes \bar{v}_{4} \otimes v_{4} \otimes a d^{k-2}\left(\left[v_{4}, \bar{v}_{4}\right]\right)\left(v_{3}\right)\right) \\
= & \beta_{2 k+1 *}\left(\left[\left[v_{4}, \bar{v}_{4}\right], v_{4}\right] \otimes \bar{v}_{4} \otimes a d^{k-2}\left(\left[v_{4}, \bar{v}_{4}\right]\right)\left(v_{3}\right)\right) \\
& +\beta_{2 k+1 *}\left(\left[\left[v_{4}, \bar{v}_{4}\right], \bar{v}_{4}\right] \otimes v_{4} \otimes a d^{k-2}\left(\left[v_{4}, \bar{v}_{4}\right]\right)\left(v_{3}\right)\right) \\
= & 0 . \quad(\text { by (ii) of Claim 1) }
\end{aligned}
$$

Hence

$$
\begin{aligned}
& \phi_{k *}\left(\iota_{4}^{\otimes 2 k} \otimes v_{3}\right) \\
&=\beta_{2 k+1 *}\left(\phi_{1} \wedge i d\right)_{*}\left(\iota_{4}^{\otimes 2} \otimes a d^{k-2}\left(\left[v_{4}, \bar{v}_{4}\right]\right)\left(v_{3}\right) \otimes\left[v_{4}, \bar{v}_{4}\right]\right) \\
&=\beta_{2 k+1 *}\left(\phi_{1} \wedge i d\right)_{*}\left(\iota_{4}^{\otimes 2} \otimes\left[v_{4}, \bar{v}_{4}\right] \otimes a d^{k-3}\left(\left[v_{4}, \bar{v}_{4}\right]\right)\left(v_{3}\right) \otimes\left[v_{4}, \bar{v}_{4}\right]\right) \\
&+\beta_{2 k+1 *}\left(\phi_{1} \wedge i d\right)_{*}\left(\iota_{4}^{\otimes 2} \otimes a d^{k-3}\left(\left[v_{4}, \bar{v}_{4}\right]\right)\left(v_{3}\right) \otimes\left[v_{4}, \bar{v}_{4}\right]^{\otimes 2}\right) \\
&= \beta_{2 k+1 *}\left(\phi_{1} \wedge i d\right)_{*}\left(\iota_{4}^{\otimes 2} \otimes a d^{k-3}\left(\left[v_{4}, \bar{v}_{4}\right]\right)\left(v_{3}\right) \otimes\left[v_{4}, \bar{v}_{4}\right]^{\otimes 2}\right) \quad(\text { as Observation A }) \\
&= \cdots
\end{aligned}
$$




$$
\begin{aligned}
= & \beta_{2 k+1 *}\left(\phi_{1} \wedge i d\right)_{*}\left(\iota_{4}^{\otimes 2} \otimes a d\left(\left[v_{4}, \bar{v}_{4}\right]\right)\left(v_{3}\right) \otimes\left[v_{4}, \bar{v}_{4}\right]^{\otimes k-2}\right) \\
= & \beta_{2 k+1 *}\left(\phi_{1} \wedge i d\right)_{*}\left(\iota_{4}^{\otimes 2} \otimes\left[v_{4}, \bar{v}_{4}\right] \otimes v_{3} \otimes\left[v_{4}, \bar{v}_{4}\right]^{\otimes k-2}\right) \\
& +\beta_{2 k+1 *}\left(\phi_{1} \wedge i d\right)_{*}\left(\iota_{4}^{\otimes 2} \otimes v_{3} \otimes\left[v_{4}, \bar{v}_{4}\right]^{\otimes k-1}\right) \\
= & \beta_{2 k+1 *}\left(\left[\left[v_{4}, \bar{v}_{4}\right], v_{3}\right] \otimes\left[v_{4}, \bar{v}_{4}\right]^{\otimes k-1}\right) \quad(\text { as Observation A) } \\
= & a d^{k}\left(\left[v_{4}, \bar{v}_{4}\right]\right)\left(v_{3}\right) . \quad(\text { by Claim 2) }
\end{aligned}
$$

This finishes the proof of Claim 3.

Claim 4. $\varphi_{k *}\left(\left[v_{4}, \bar{v}_{4}\right] \otimes a d^{k-1}\left(\left[v_{4}, \bar{v}_{4}\right]\right)\left(v_{3}\right)\right)=0$.

Proof of Claim 4. Note that

$$
\begin{aligned}
\varphi_{k} & =\left(i d \wedge \varphi_{1}\right)\left(\varphi_{k-1} \wedge i d\right) \\
& =\left(i d \wedge \varphi_{1}\right)\left(i d \wedge \varphi_{1} \wedge i d\right)\left(\varphi_{k-2} \wedge i d\right) \\
& =\cdots \\
& =\left(i d \wedge \varphi_{1}\right) \cdots\left(\varphi_{1} \wedge i d\right) \\
& =\left(i d \wedge \varphi_{1}\right) \cdots\left(\left(\varphi_{1} \beta_{3}\right) \wedge i d\right) \quad\left(\text { note that } \varphi_{1} \beta_{3}=\varphi_{1}\right)
\end{aligned}
$$

By (i) of Claim 1, we have $\varphi_{k *}([x, y] \otimes \cdots)=0$.

This finishes the proof of Claim 4.

Now we have

$$
\begin{aligned}
\varphi_{k *} \phi_{k *}\left(\iota_{4}^{\otimes 2 k}\right. & \left.\otimes v_{3}\right) \\
& =\varphi_{k *}\left(a d^{k}\left(\left[v_{4}, \bar{v}_{4}\right]\right)\left(v_{3}\right)\right) \\
& =\varphi_{k *}\left(\left[v_{4}, \bar{v}_{4}\right] \otimes a d^{k-1}\left(\left[v_{4}, \bar{v}_{4}\right]\right)\left(v_{3}\right)\right)+\varphi_{k *}\left(a d^{k-1}\left(\left[v_{4}, \bar{v}_{4}\right]\right)\left(v_{3}\right) \otimes\left[v_{4}, \bar{v}_{4}\right]\right) \\
& =\varphi_{k *}\left(a d^{k-1}\left(\left[v_{4}, \bar{v}_{4}\right]\right)\left(v_{3}\right) \otimes\left[v_{4}, \bar{v}_{4}\right]\right) \quad(\text { by Claim 4) } \\
& =\left(i d \wedge \varphi_{1}\right)_{*}\left(\varphi_{k-1} \wedge i d\right)_{*}\left(a d^{k-1}\left(\left[v_{4}, \bar{v}_{4}\right]\right)\left(v_{3}\right) \otimes\left[v_{4}, \bar{v}_{4}\right]\right) \\
& =\left(i d \wedge \varphi_{1}\right)_{*}\left(\varphi_{k-1 *}\left(a d^{k-1}\left(\left[v_{4}, \bar{v}_{4}\right]\right)\left(v_{3}\right)\right) \otimes\left[v_{4}, \bar{v}_{4}\right]\right) \\
& =\left(i d \otimes \varphi_{1 *}\right)\left(\varphi_{k-1 *}\left(\phi_{k-1 *}\left(\iota_{4}^{\otimes 2 k-2} \otimes v_{3}\right)\right) \otimes\left[v_{4}, \bar{v}_{4}\right]\right) \quad \text { (by Claim 3) } \\
& =\left(i d \otimes \varphi_{1 *}\right)\left(\iota_{4}^{\otimes 2 k-2} \otimes v_{3} \otimes\left[v_{4}, \bar{v}_{4}\right]\right) \quad(\text { by inductive condition }) \\
& =\iota_{4}^{\otimes 2 k-2} \otimes \varphi_{1 *}\left(v_{3} \otimes\left[v_{4}, \bar{v}_{4}\right]\right) \\
& =\iota_{4}^{\otimes 2 k-2} \otimes \varphi_{1 *}\left(\beta_{3 *}\left(v_{3} \otimes\left[v_{4}, \bar{v}_{4}\right]\right)\right) \quad\left(\text { note that } \varphi_{1} \beta_{3}=\varphi_{1}\right) \\
& =\iota_{4}^{\otimes 2 k-2} \otimes \varphi_{1 *}\left(\left[\left[v_{4}, \bar{v}_{4}\right], v_{3}\right]\right) \quad(\text { by }(\mathrm{i}) \text { of Claim 1) } \\
& =\iota_{4}^{\otimes 2 k-2} \otimes \varphi_{1 *}\left(\phi_{1 *}\left(\iota_{4}^{\otimes 2} \otimes v_{3}\right)\right) \\
& =\iota_{4}^{\otimes 2 k} \otimes v_{3} .
\end{aligned}
$$

Therefore, we complete the proof of Lemma 4.1.

\section{Proof of Theorem 1.1 and Corollary 1.2}

The following Proposition 5.1 is easily obtained from Theorem 1.6 of [14].

Proposition 5.1. Let $X=\Sigma X^{\prime}$ be a path-connected 2-local finite $C W$-complex. Let $2<k_{1}<k_{2}<\cdots$ be a sequence of odd integers such that no $k_{j}$ is a multiple of any 
other. Then there exists a topological space $A$ such that

$$
\Omega \Sigma X \simeq \prod_{j} \Omega \Sigma L_{k_{j}}(X) \times A
$$

localized at 2 .

Proof of Theorem 1.1 for $n=3$. By Proposition 2.1 and the Hilton-Milnor Theorem [13], we get

$$
\Omega \Sigma L_{k_{j}}\left(C_{r}^{5, r}\right) \simeq \Omega \Sigma C_{r}^{4 k_{j}+1, r} \times(\text { some other spaces }) .
$$

Hence Theorem 1.1 is easily obtained from Proposition 5.1.

Proof of Corollary 1.2 for $n=3 . \pi_{m}\left(\Sigma C_{r}^{4 p+1, r}\right)$ is a summand of $\pi_{m}\left(C_{r}^{6, r}\right)$ for any odd integer $p \geqslant 3$ from Theorem 1.1 .

Let $p$ be large enough such that $p \geqslant \frac{k-2}{4}$. By the Freudenthal suspension theorem,

$$
\pi_{k+4 p-4}\left(\Sigma C_{r}^{4 p+1, r}\right) \cong \pi_{k+4 p-4}^{s}\left(\Sigma C_{r}^{4 p+1, r}\right) \cong \pi_{k}^{s}\left(C_{r}^{6, r}\right) .
$$

Therefore, $\pi_{k}^{s}\left(C_{r}^{6, r}\right)$ is a summand of $\pi_{k+4 p-4}\left(C_{r}^{6, r}\right)$.

\section{References}

[1] P. Beben and J. Wu, Modular representations and the homotopy of low rank p-local CW complexes, Math. Z. 273 (2013), 735-751.

[2] D.J. Benson, Representations and cohomology I: Basic representation theory of finite groups and associative algebras, Bull. Lond. Math. Soc. 26(6) (1994), $485-485$.

[3] S.C. Chang, Homotopy invariants and continuous mappings, Proc. R. Soc. Lond. Ser. A 202 (1950), 253-263.

[4] D.W Chen and J. Wu, Decomposition of loop spaces and periodic problem on $\pi_{*}$, Algebr. Geom. Topol. 13 (2013), 3245-3260.

[5] F.R Cohen, Applications of loop spaces to some problems in topology, London Math. Soc. Lecture Note Ser. 139 (1989), 10-20.

[6] F.R. Cohen, J.C. Moore, and J.A. Neisendorfer, Torsion in homotopy groups, Ann. of Math. 109 (1979), 121-168.

[7] F.R. Cohen and J. Wu, A remark on the homotopy groups of $\Sigma^{n} \mathbb{R} P^{2}$, Contemp. Math. 181 (1995), 65-81.

[8] S. Priddy, Lectures on the stable homotopy of BG, Geom. Topol. Monogr. 11 (2007), 289-308.

[9] P. Selick and J. Wu, On natural decompositions of natural coalgebra and loop suspensions, Mem. Amer. Math. Soc. 148 (2000), no. 701.

[10] P. Selick and J. Wu, On functorial decompositions of self-smash products, Manuscripta Math. 111 (2003), 435-457.

[11] P. Selick and J. Wu, The functor $A^{\text {min }}$ on p-local spaces, Math. Z. 253 (2006), 435-451. 
[12] J.P. Serre, Homologie singuliére des espaces fibrés, Applications. Ann. of Math. (2) 54 (1951), 425-505.

[13] G.W. Whitehead, Elements of homotopy theory, Springer-Verlag, 97 (1978).

[14] J. Wu, On combinatorial calculations for the James Hopf maps, Topology 37 (1998), 1011-1023.

[15] J. Wu, Homotopy theory of the suspensions of the projective plane, Mem. Amer. Math. Soc. 162 (2003), no. 769.

[16] Z.J. Zhu and J.Z. Pan, The decomposability of smash product of $A_{n}^{2}$ complexes, Homology Homotopy Appl. 19 (2017), 293-318.

Zhongjian Zhu zhuzhongjian@amss.ac.cn

College of Mathematics, Physics and Electronic Information Engineering, Wenzhou University, Wenzhou, Zhejiang 325035, China

Pengcheng Li xiaotianshishou@163.com

Academy of Mathematics and Systems Science, Chinese Academy of Sciences; University of Chinese Academy of Sciences, Beijing, 100190, China

Jianzhong Pan pjz@amss.ac.cn

Hua Loo-Keng Key Mathematical Laboratory, Institute of Mathematics, Academy of Mathematics and Systems Science, Chinese Academy of Sciences; University of Chinese Academy of Sciences, Beijing, 100190, China 